\title{
A Dual Tabu Search Algorithm for Vehicle Routing Problem
}

\author{
Tao Wang ${ }^{1, \text { a }}$ \\ ${ }^{1}$ Network Information Center, Tonghua Normal University, TongHua, Jilin, China \\ anash_k127@126.com
}

Keywords: Vehicle Routing Problem; Tabu Search Algorithm; Double Tabu Search Algorithm.

\begin{abstract}
In this paper, vehicle routing problem as the background, the traditional tabu search algorithm is proposed based on a set of methods to build the initial solution and its integration to achieve the dual tabu search algorithm for vehicle routing problem. A new algorithm to avoid the shortcomings of the traditional tabu search algorithm is too dependent on the initial solution, the simulation tests to verify the feasibility and effectiveness of the algorithm.
\end{abstract}

\section{Introduction}

Vehicle Routing Problem (hereinafter referred to as VRP) has been proven to be NP-hard, so now to solve this problem algorithm focused on approximation algorithms, common algorithms are: tabu search algorithm, genetic algorithm, ant colony algorithm. Tabu search algorithm which have "climbing" ability, searching high efficiency, but the algorithm is a strong dependence on the initial solution, especially large-scale VRP problem, select the initial solution to obtain a final "optimal solution "Greater impact. Aiming at this shortcoming tabu search algorithm, the idea of a double taboo search in the selected initial solution also uses the principle of tabu search, the establishment of a set of initial solution, the initial solution set theory algorithms to avoid the follow-up into a "local optimum excellent ", thereby circumventing the defect tabu search algorithm to improve the algorithm of global optimization, optimal strategies for exploring the VRP provides a new way of thinking. The simulation results show the feasibility and effectiveness of the algorithm.

\section{Dual Tabu Search Algorithm}

Solution Set initial construction method. Traditional tabu search algorithm start from an initial solution through the internal mechanism of the algorithm, after several iterations to obtain the optimal solution. But the algorithm is much higher dependence on initial solution, hence, we propose a method for the construction of an initial set of solutions to reduce the traditional tabu search algorithm, the initial solution dependencies.

Since the method of constructing the neighborhood is to generate new solutions exchange position solution adjacent elements, so the difference in the neighborhood of the method constructed between SOLUTIONS is mainly reflected in the "public side" (the solution in the same or a similar path that is public side), the neighborhood solution concentrated solutions mostly have a lot of items of "public side." As a starting point, this article needs to build the initial solution concentration should be made public as little as possible between the edges of different solutions, so this paper, a method to obtain a taboo to build the initial solution set.

The method is as follows:

We randomly generate an initial solution, encoding solution as: Let the customer set is $C=\{1,2, \cdots, n\}$, the total amount of customer and logistics center is $N=\{0,1, \cdots, n\}$, Where 0 represents the logistics center, logistics center has $k$ cars of the same model car, we insert 0 in the collection, the count is $k-1$, we know $X=\{0,1,2, \ldots, n, n+1, \ldots n+(k-1)\}$, Random permutation is the initial solution.

In the initial solution in the first element, starting with 0 for each element added tabu list, elements of the elements in each of the initial solution in behind the into the taboo table, where 0 can be added more than once. When the tabu list length equal to the solution of a count of the number of elements, 
release tabu list in the first to join the elements. Then randomly selected in a non tabu list elements are added later. According to this method eventually produce new initial solution.

Taking into account the computation time, the number of initial solution concentration should be no more than 10 , that is, the termination criterion of the tabu search algorithm for generating the initial solution set is: the number of $\mathrm{R}$ is less than or equal to 10

\section{2 double tabu search algorithm design}

Construction of the initial solution set, in theory, to the traditional tabu search algorithm provides a way to override the global might, on this basis, for each element of the solution set of tabu search algorithm to obtain a provisional optimal solution, and finally horizontal comparison, the best target is the global optimal solution.

The overall implementation of the algorithm is as follows:

the use of previously taboo proposed acquisition algorithm to generate the initial solution set. each element of the initial solution set as input, respectively search.

Given an initial solution $x^{*}$, the objective function value $Z^{*}=z\left(x^{*}\right)$. So that the current solution $x=x^{*}$, set tabu list is empty, and the length of the taboo $L=\eta$. Cycle start:

While (termination criterion: given a number of iterations)

if (iterations $==0$, the current solution $x=x^{*}$ )

Construction of the initial solution neighborhood;

Generating a candidate solution set;

From the selection of candidate solutions focus taboo subject, and the next iteration of the current solution;

else if (Tabu length $L>=\eta$ )

The extended table taboo taboo object releases;

Construction of the current solution neighborhood;

Generating a candidate solution set;

Update tabu list, we focused on the selection of candidates for a solution as the new current solution;

else

Construction of the current solution neighborhood;

Generating a candidate solution set;

Added directly taboo subject, and pick the candidate solution set as the new current solution; Plus a number of iterations;

End of the cycle.

Where $z(x)$ is the objective function for the VRP. The proposed algorithm after a given initial solution into the loop, the loop body has three options determine: in (4) When the cycle starts, the current solution is initialized to the initial solution, tabu table is empty, execution neighborhood search tasks. In (5) If the length is greater than or equal to the nominal contraindicated length, extended release taboo object, and then execute the neighborhood search task. In (6) If the length is less than the nominal length of the taboo, the direct implementation of neighborhood search tasks. The end of the first iteration, plus a local variable, enter the next cycle.

$x^{*}$ is the current optimal solution for $Z^{*}$ is the temporary optimum value of searching. Enter the initial solution set at an initial solution, the process is repeated until the initial solution concentration of the solution of all the search is completed.

Results: temporary optimum value, whichever is the best search algorithm as to the optimal value, the corresponding solution as optimal output.

\section{Simulation}

In this paper, the experimental environment is: CPU: Intel (R) Xeon (R) 3.30GHz; RAM: DDR3 8.00GB; Development Tools: Java; development environment: MyEclipse 8.5; OS: Windows7. 
Example: Suppose a logistics center with 5 cars of the same type, the number of customers is 19, its coordinates and requirements are shown in table 1:

Table 1 Customer coordinate and demand

\begin{tabular}{cccc||cccc}
\hline $\begin{array}{c}\text { Customer } \\
\text { ID }\end{array}$ & $\begin{array}{c}\text { Abscissa } \\
\text { x/km }\end{array}$ & $\begin{array}{c}\text { Y-axis } \\
\mathrm{y} / \mathrm{km}\end{array}$ & $\begin{array}{c}\text { Demand } \\
\mathrm{g} / \mathrm{t}\end{array}$ & $\begin{array}{c}\text { Customer } \\
\text { ID }\end{array}$ & $\begin{array}{c}\text { Abscissa } \\
\mathrm{x} / \mathrm{km}\end{array}$ & $\begin{array}{c}\text { Y-axis } \\
\mathrm{y} / \mathrm{km}\end{array}$ & $\begin{array}{c}\text { Demand } \\
\mathrm{g} / \mathrm{t}\end{array}$ \\
\hline 0 & 0 & 0 & 0 & 10 & 1 & 3 & 0.6 \\
1 & 0 & -1 & 1.5 & 11 & 3 & 4 & 0.2 \\
2 & 0 & 3 & 1.8 & 12 & -3 & 0 & 2.4 \\
3 & -2 & -2 & 2.0 & 13 & 2 & 0 & 1.9 \\
4 & -3 & -3 & 0.8 & 14 & 1 & -3 & 2.0 \\
5 & 3 & -1 & 1.5 & 15 & 2 & -1 & 0.7 \\
6 & -4 & 0 & 1.0 & 16 & 2 & 1 & 0.5 \\
7 & -4 & -1 & 2.5 & 17 & 1 & -4 & 2.2 \\
8 & 1 & -2 & 3.0 & 18 & -3 & 2 & 3.1 \\
9 & 1 & -1 & 1.7 & 19 & -1 & -1 & 0.1
\end{tabular}

Set the initial parameters are: Tabu list length is 5 , the number of neighborhood is 50 , terminate iterations 500, the objective function value represents the total distance. The results are: the optimal target $=73.1$, using the number of vehicles: 4 , computation time: $1.6 \mathrm{~S}$.

The optimal solution is $\{0,18,7,15,8,6,10,11,0,19,14,9,2,5,13,17,0,12,16,0,3,1,4,0\}$.

To further test the dual effect of tabu search algorithm calculations, we use the VRP common international study library: (VRP LIB (http://www.branchandcut.org/) in the E-n51-k5.vrp with other algorithms Compare.

Table 2 E-n51-k5.vrp Comparison of the optimal values of each algorithm

\begin{tabular}{lcccccc}
\hline Algorithm & DM & TS & GA & ACO & PS & DoubleTS \\
\hline Best solution & 524 & 524.61 & 524.8 & 524.61 & 524.61 & 522.76 \\
Difference & 3 & 3.61 & 3.8 & 3.61 & 3.61 & 1.76 \\
\hline
\end{tabular}

As can be seen from Table 2, the optimal target double tabu search algorithm is superior to the results obtained by other algorithms to prove the proposed algorithm, the feasibility, effectiveness and good optimization ability.

\section{Conclusion}

Double tabu search algorithm proposed by simulation experiments, to obtain a more satisfactory result. Hence, The traditional tabu search algorithm is feasible for improvement. The double tabu search algorithm to search the basic framework of the traditional algorithm based on tabu search, using a tabu search acquisition algorithm, to obtain an initial solution set, the solution set theory to cover the global, to avoid the defects of the traditional tabu search algorithm is too dependent on the initial solution from the experiment. Test example can be seen in the double tabu search algorithm compared with other algorithms, the optimal solution of the higher quality, especially in the process of solving large scale problems is more obvious. However, due to the construction of the initial solution set tabu acquisition algorithm is too complicated, and only if the programming level is limited, the computation time is slightly longer that need to be further explored.

\section{References}

[1] Glover F. Tabu Search: partII[J]. ORSA Journal on Computing, 1990,2:4-32.

[2] Glover F. Tabu Search: partI[J].ORSA Journal on Computing, 1989,1:190-260.

[3] William J. Cook, William H. Cunningham, William R. Pulleyblank, Alexander Schrijver. combinatorial optimization[M] New York: John Wiley \& Sons,1998. 
[4] Glover F. A Template for Scatter Search and Path Relinking [C]In: Hao, J K, Lutton E, Ronald E, Schoenauer M, Snyers D. (Eds.).Artificial Evolution, Lecture Notes in Computer Science (J0302-9743), 1998, 1363. Germany: Springer, 1998: 13-54.

[5] Paolo Toth,Daniele Vgo[M].The Vehicle Routing Problem ,2002.

[6] Balinski M, Quand R. Cn an integer program for a delivery problem[J]. Operations R,esearch, 1962, 12: 300-204.

[7] Teodorovic D, Pavkovic G. A Simulated Annealing Technique Approach to the Vehicle Routing Problem in the Case of Stochastic Demand[J]. Transportation Planning and Technology. 1992, 15: 261-273.

[8] Psaraftis. H. A Dynamic Programming Solution to the Single Vehicle Many - to-Many Immediate Request Dial Ride Problem[J]. Transportation Science.1980.14:130-154. 